\title{
Epilepsy-causing sequence variations in SIK1 disrupt synaptic activity response gene expression and affect neuronal morphology
}

\author{
Christoph Pröschel ${ }^{1}$, Jeanne N Hansen ${ }^{2,3}$, Adil Ali $^{3}$, Emily Tuttle ${ }^{3}$, Michelle Lacagnina ${ }^{1}$, Georgia Buscaglia ${ }^{1}$, \\ Marc W Halterman ${ }^{3,4}$ and Alex R Paciorkowski ${ }^{\star, 1,2,3,4}$
}

SIK1 syndrome is a newly described developmental epilepsy disorder caused by heterozygous mutations in the salt-inducible kinase SIK1. To better understand the pathophysiology of SIK1 syndrome, we studied the effects of SIK1 pathogenic sequence variations in human neurons. Primary human fetal cortical neurons were transfected with a lentiviral vector to overexpress wildtype and mutant SIK1 protein. We evaluated the transcriptional activity of known downstream gene targets in neurons expressing mutant SIK1 compared with wild type. We then assayed neuronal morphology by measuring neurite length, number and branching. Truncating SIK1 sequence variations were associated with abnormal MEF2C transcriptional activity and decreased MEF2C protein levels. Epilepsy-causing SIK1 sequence variations were associated with significantly decreased expression of $A R C$ (activity-regulated cytoskeletal-associated) and other synaptic activity response element genes. Assay of mRNA levels for other MEF2C target genes NR4A1 (Nur77) and NRG1, found significantly, decreased the expression of these genes as well. The missense p.(Pro287Thr) SIK1 sequence variation was associated with abnormal neuronal morphology, with significant decreases in mean neurite length, mean number of neurites and a significant increase in proximal branches compared with wild type. Epilepsy-causing SIK1 sequence variations resulted in abnormalities in the MEF2C-ARC pathway of neuronal development and synapse activity response. This work provides the first insights into the mechanisms of pathogenesis in SIK1 syndrome, and extends the ARX-MEF2C pathway in the pathogenesis of developmental epilepsy.

European Journal of Human Genetics (2017) 25, 216-221; doi:10.1038/ejhg.2016.145; published online 14 December 2016

\section{INTRODUCTION}

SIK1 syndrome is a newly described developmental epilepsy disorder (OMIM no. 616341) caused by de novo pathogenic sequence variations in the salt-inducible kinase SIK1. ${ }^{1}$ SIK1 is an AMP family member kinase $^{2}$ and regulator of CREB1 and MEF2C transcriptional activity. ${ }^{3}$ Loss-of function sequence variations of $M E F 2 C$ are also a cause of developmental epilepsy and intellectual disability. ${ }^{4-6}$ Several genes have been shown to be jointly regulated by MEF2C and CREB1 through the synaptic activity response element mechanism. ${ }^{7}$ Of additional interest is that $M E F 2 C$ has also been shown to be regulated by $A R X$ transcriptional activity, ${ }^{8}$ which is relevant because sequence variations in $A R X$ were among the earliest demonstrated genetic cause of developmental epilepsy. ${ }^{9}$ The discovery of SIK1 syndrome extends the $A R X-M E F 2 C$ pathway and is an opportunity to increase our understanding of a class of developmental epilepsy syndromes.

Although an increasing number of genetic causes of early-life epilepsy have been identified, the molecular mechanisms whereby sequence variations lead to disease remain poorly understood. In some cases, understanding of the genetic cause of epilepsy has resulted in the selection of appropriate antiseizure therapy, ${ }^{10,11}$ or consideration of novel therapies. ${ }^{12,13}$ Therefore, studies to understand how pathogenic sequence variations in a specific gene lead to disease will be critical in improving the care of patients with epilepsy. To better characterize the pathophysiology of SIK1-related epilepsy, we studied the effects of epilepsy-causing SIK1 sequence variations in human fetal neurons.

\section{MATERIALS AND METHODS}

Epilepsy-causing SIK1 sequence variations

We studied pathogenic variants in SIK1 that have been submitted to the ClinVar database (Supplementary Table 1).

Lentiviral delivery of SIK1 mutant proteins

To allow stable expression of mutant SIK1 proteins, cDNA encoding wild-type SIK1 (NM_173354.3) and the mutants p.(Pro287Thr), p.(Glu347*), p. $\left(\mathrm{Gln} 633^{\star}\right)$ and p.(Lys56Met; a kinase-dead control) were cloned into the Xbal and Notl sites of the lentiviral vector pCDH-GFP-Puro (CD513B; System Biosciences, Palo Alto, CA, USA). Details of codon modifications based on SIK1 protein ID NP_775490.2 used for cloning are provided in Supplementary Table 2. To identify recombinant SIK1 protein expression, a V5-epitope tag was inserted in-frame at the amino terminal of the SIK1 cDNA. Vector controls included pCDH-GFP-Puro without insert or expressing the nuclear envelope protein lamin A. Amphotrophic lentivirus was produced in HEK293-TN cells using the VSVg envelope protein, and virus was harvested in serum-free medium. Expression of mutant proteins was verified by western blot analysis of HEK293-TN cell lysates using anti-V5 antibody (Bethyl Laboratories, Montgomery, TX, USA) and immunofluorescence staining of transfected cells with anti-V5.

${ }^{1}$ Department of Biomedical Genetics, University of Rochester Medical Center, Rochester, NY, USA; ${ }^{2}$ Department of Pediatrics, University of Rochester Medical Center, Rochester, NY, USA; ${ }^{3}$ Center for Neural Development and Disease, University of Rochester Medical Center, Rochester, NY, USA; ${ }^{4}$ Departments of Neurology and Neuroscience, University of Rochester Medical Center, Rochester, NY, USA

${ }^{*}$ Correspondence: Dr AR Paciorkowski, Department of Child Neurology, University of Rochester Medical Center, 601 Elmwood Avenue, Rochester, NY 14642, USA. Tel: +1 585275 2808; Fax: +1 585275 3683; E-mail: Alex_Paciorkowski@urmc.rochester.edu

Received 7 March 2016; revised 20 September 2016; accepted 27 September 2016; published online 14 December 2016 


\section{Isolation of human fetal neurons}

Primary human cortical neurons were isolated from CNS tissue obtained from cadavers at 14-20 weeks of gestation. No personal identifiers or medical history was provided. However, samples with known genetic abnormalities or HIV+ status were excluded from tissue collection. As per certification by the University of Rochester Research Subjects Review Board (RSRB 24759) this work does not constitute human subject research. Tissues were processed as described previously. ${ }^{14,15}$ Briefly, following enzymatic and mechanical dissociation, debris was removed from the cell suspension through sequential filtering and density gradient centrifugation. Cells were then plated on poly-L-lysine/ laminin-coated substrate and cultured in Neurobasal medium with the addition of B27, N2, BDNF and GDNF. Cells were maintained in low oxygen atmosphere $\left(5 \% \mathrm{O}_{2}\right)$ with $50 \%$ medium exchange every other day.

\section{Transduction and selection of neural cultures}

Primary neural cultures were incubated for up to $6 \mathrm{~h}$ with lentivirus containing supernatants. Two days after infection, cultures underwent puromycin selection by the addition of $2 \mu \mathrm{g} / \mathrm{ml}$ puromycin for 3 days, a dosage that successfully eliminated all puromycin-sensitive cells. Complete selection of transduced cells was monitored by the expression of GFP in all cells.

\section{Mef2 and CREB luciferase reporter assays}

We used a previously characterized Mef2 luciferase reporter ${ }^{16}$ to assay the effect of SIK1 pathogenic sequence variations on Mef2c-based transcription. Briefly, 293TN cells were transfected simultaneously with a SIK1 expression construct pcDNA3.1Mef2c-HA (plasmid no. 32515 from Addgene, Cambridge, MA, USA), 3xMef2Luc (plasmid no. 32976) and pRL-TK (Promega, Madison, WI, USA). The next day, firefly and Renilla luciferase activity was measured using Promega's Dual-Glo Luciferase Assay System and a SpectraMax M5 microplate reader (Molecular Devices, Sunnyvale, CA, USA). To measure CREB activity, the pCRE-MetLuc2 vector (Clontech, Mountain View, CA, USA) and our SIK1 expression vectors were transfected into 293TN cells. The day after transfection, basal luciferase expression was measured $3 \mathrm{~h}$ after a full media change using a Ready-To-Glow Secreted Luciferase Reporter Assay (Clontech). A second full media change was used to start the Forskolin/IBMX treatment. Aliquots of media were collected at 1,3 and $5 \mathrm{~h}$ to assay for luciferase expression during drug treatment.

\section{Semiquantitative reverse transcriptase-PCR}

Total mRNA was collected from transfected human neurons after 15 days of culture (Qiagen RNeasy Mini Kit, Qiagen, Hilden, Germany). cDNA was then prepared using the iScript cDNA Synthesis Kit (Bio-Rad, Hercules, CA, USA). Semiquantitative reverse transcriptase-PCR (RT-PCR) was performed using validated TaqMan probes to determine the relative expression levels of known $M E F 2 C$ target genes ARC (activity-regulated cytoskeletal-associated), NRG1 and NR4A1 (Nur77). Expression of housekeeping gene UBC was used for Delta-Delta analysis of relative gene expression. TaqMan probes used in these assays are provided in Supplementary Table 3.

\section{Western blots}

Transfected neurons were lysed in RIPA buffer ( $25 \mathrm{~mm}$ Tris-Cl, pH 7.6, $150 \mathrm{~mm}$ $\mathrm{NaCl}, 1 \%$ Triton X-100, 0.5\% DOC, $0.1 \%$ SDS) with Proteogard protease inhibitor (Clontech) and sonicated. Lysates were separated by SDS-PAGE. Proteins were transferred onto PVDF membrane for western blots. Membranes were blocked with 5\% NFDM (non-fat dry milk) in TBS-T solution and probed with a goat anti-mouse-HRP secondary antibody (Santa Cruz Biotechnology, Santa Cruz, CA, USA). HRP signal was visualized using Western Lightning-ECL (Perkin-Elmer, Waltham, MA, USA) and Amersham Hyperfilm (GE Healthcare, Little Chalfont, UK). Antibodies used in western blots are provided in Supplementary Table 4.

\section{Neuronal morphology studies}

Primary cortical neurons transduced with control and mutant SIK1 protein expressing lentivirus were fixed after 3 days in vitro and stained for $\beta$ III-tubulin. Digital images were collected under x20 magnification. Dendrite number and length were measured and Scholl analyses were performed for dendritic arborization in 50 cells per condition using the ImageJ software (http://imagej.nih.gov/ij/) and the Fiji/Simple Neurite Tracer plugin..$^{17,18}$ Blinding of samples was performed by a third party before morphology studies.

\section{Statistical analysis}

Statistical analysis was performed with $\mathrm{R}$ version 3.1.2 (https://www.r-project. org/) and $P$-values $<0.05$ were regarded as significant.

\section{RESULTS}

Truncating SIK1 pathogenic sequence variations were associated with abnormal MEF2C transcriptional activity and decreased MEF2C protein levels

Using a lentiviral vector, we infected human primary cortical neurons with a V5-epitope-tagged wild-type or mutant SIK1 (Figure 1a), and achieved robust expression as detected by western blot (Figure 1b) and immunocytochemistry (Figures 1c-e). The levels of endogenous SIK1 in the mutant cell lines did not differ significantly from that found in the lamin or WT overexpression cell lines (Supplementary Figure 1). In nonneuronal cells, we measured the level of MEF2 transcriptional activity using a characterized Mef2 luciferase reporter in the presence of epilepsycausing SIK1 pathogenic sequence variations, as well as wild-type and the p.(Lys56Met) kinase-dead mutant. As expected, the kinase-dead mutant showed no significant Mef2-luc activity compared with empty vector controls. Expression of wild-type SIK1 robustly activated the Mef2-luc reporter. In non-neuronal cells, epilepsy-causing SIK1 mutants were associated with a significant decrease in Mef2-luc reporter expression compared with wild-type (data not shown). We therefore sought to expand these findings in our neuronal model of SIK1 pathogenic sequence variations. We suspected that epilepsy-causing sequence variations in SIK1 might, in part, exert their pathogenic effect through disruption of MEF2C transcriptional activity.

MEF2 proteins regulate their own transcription via a conserved enhancer in muscle cells and brain, ${ }^{19}$ with evidence for regulational crosstalk between MEF2A and MEF2C. ${ }^{20}$ To investigate the effects of SIK1 pathogenic sequence variations on MEF2C transcriptional activity, we first assayed the protein levels of MEF2C itself. We found that in neurons expressing the epilepsy-causing SIK1-truncating sequence variations p. (Glu347*) and p. $\left(\mathrm{Gln} 633^{\star}\right)$, there were decreased MEF2C protein levels compared with wild type as measured by western blot (Figure 2). Interestingly, this effect on MEF2C protein levels was not seen in neurons expressing the missense SIK1 sequence variation p.(Pro287Thr).

Epilepsy-causing SIK1 sequence variations were associated with decreased expression of $A R C$ and other synaptic activity response element genes

Using mRNA and protein assays, we found that neurons expressing epilepsy-causing SIK1 sequence variations had significantly decreased mRNA levels of the gene $A R C$ (Figure 3a), compared with neurons expressing wild-type SIK1. We confirmed that significantly decreased protein levels of $A R C$ occurred in neurons at 20 weeks gestation that expressed the epilepsy-causing truncating SIK1 sequence variations p.(Glu347*) and p.(Gln633*; Figure 3b), confirmed by western blot (Figures $3 \mathrm{c}-\mathrm{e})$. While the missense SIK1 sequence variation $\mathrm{p}$. (Pro287Thr) was associated with significantly decreased ARC mRNA levels, decreased ARC protein levels were not found. There was a downward trend in ARC protein levels in neurons expressing the kinase-dead p.(Lys56Met) SIK1 mutant, but not significantly different from wild type.

Assay of mRNA levels for other MEF2C target genes NR4A1 (Nur77) and NRG1, found significantly, decreased expression of these 
genes in neurons at 20 weeks gestation as well. An accompanying decrease in NR4A1 and NRG1 protein levels could not be confirmed owing to the lack of antibodies that performed in western blot. These qRT-PCR results are summarized in Supplementary Figure 2.

SIK1 pathogenic sequence variations did not affect levels of phosphorylated HDAC5

We had previously observed that epilepsy-causing sequence variations in SIK1 did not affect the ability of SIK1 to phosphorylate target HDAC5 during an in vitro assay, ${ }^{1}$ but we hypothesized that the inability of truncating mutant SIK1 proteins to localize into the nucleus might functionally result in decreased HDAC5 phosphorylation. Compared with wild-type SIK1, none of the epilepsy-causing SIK1 mutants was associated with significant differences in phosphorylation of p.Ser498 compared with total HDAC5 (Supplementary Figure 3). The kinase-dead SIK1 p.(Lys56Met) mutant was associated with HDAC5 phosphorylation similar to wild type on western blot, confirming that HDAC5 is a target for phosphorylation by other kinases in vivo, and that endogenous SIK1 still can phosphorylate HDAC5 in our system. ${ }^{21}$

SIK1 pathogenic sequence variations do not affect TORC1 or CREB1 protein levels or CREB1 transcriptional activity

We did not observe a significant decrease in CREB1 transcriptional activity using an available CREB luciferase reporter assay with SIK1 mutants compared with wild type in HEK/293 cells (data not shown). Protein levels of TORC1 and CREB1 were also no different by western blot in neurons expressing SIK1 sequence variations, compared with controls (data not shown).

Truncating epilepsy-causing sequence variations in SIK1 were associated with abnormal neuronal morphology

As $A R C$ was found to be critical for dendrite spine morphology, ${ }^{22}$ we asked whether SIK1 pathogenic sequence variations are associated with abnormal morphology in vitro. We performed a Sholl analysis to quantify dendrite branching in human fetal neurons expressing wild-type and mutant SIK1 proteins. We found that the missense p.(Pro287Thr) SIK1 sequence variation was associated with significantly increased number of proximal branches. This effect was not seen in the truncating SIK1 mutants p. $($ Glu347*) and p. (Gln633*).

a
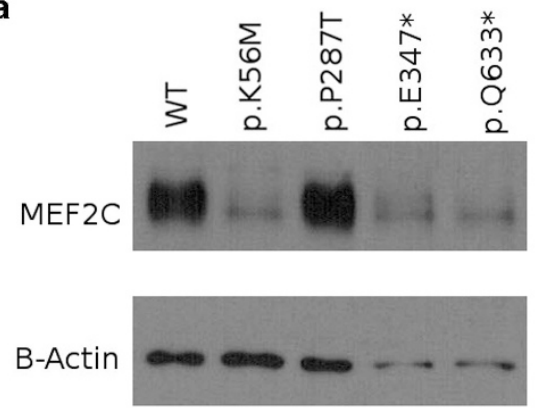

b

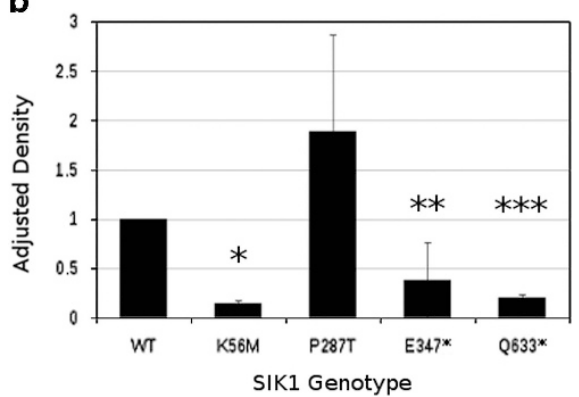

Figure 2 Truncating SIK1 sequence variations decreased MEF2C protein levels compared with wild type. (a) Representative western blot of MEF2C protein levels in human neurons at 20 weeks gestation. (b) Differential MEF2C protein levels were observed depending on SIK1 genotype. The kinase-dead p.(Lys56Met) mutant, as well as the truncating SIK1 epilepsycausing mutants p.(Glu247*) and p.(Gln633*), showed significantly reduced MEF2C protein levels normalized to $\beta$-actin. The missense SIK1 sequence variation p.(Pro287Thr) showed MEF2C protein levels similar to wild type. ${ }^{*} P=0.02,{ }^{* *} P=0.02$, and ${ }^{* *} P=0.02$.

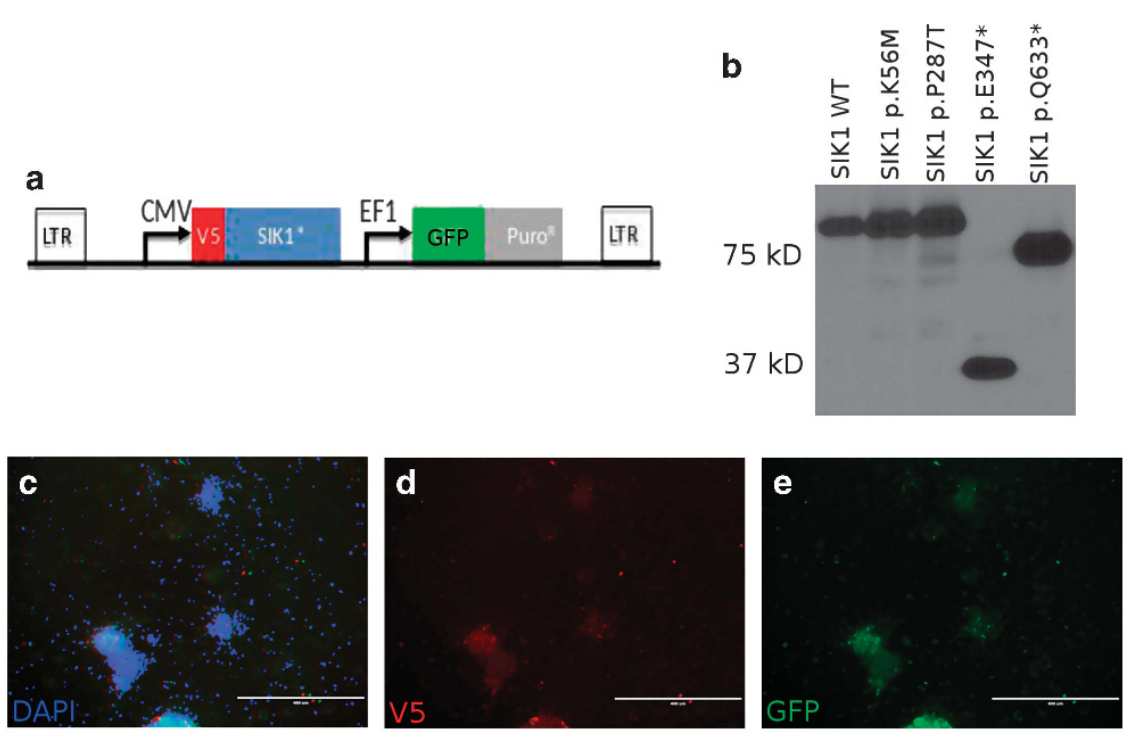

Figure 1 Expression of SIK1 wild-type and mutant proteins in human fetal neurons. (a) The lentiviral vector pCDH-GFP-Puro was used with a V5-epitope tag inserted in-frame at the amino terminal of the SIK1 CDNA. Vector controls included pCDH-GFP-Puro without insert or expressing the nuclear envelope protein lamin A. (b) Western blot of V5-tagged, wild-type and mutant SIK1 proteins in primary human neurons derived from the cortex at 20 weeks of gestation. (c-e) Example of lentiviral transduction followed by puromycin selection of primary human neurons (c) with SIK1 p.(GIn633*) resulted in robust V5 (d) and GFP staining (e). Bar $=400 \mu \mathrm{m}$. 
a

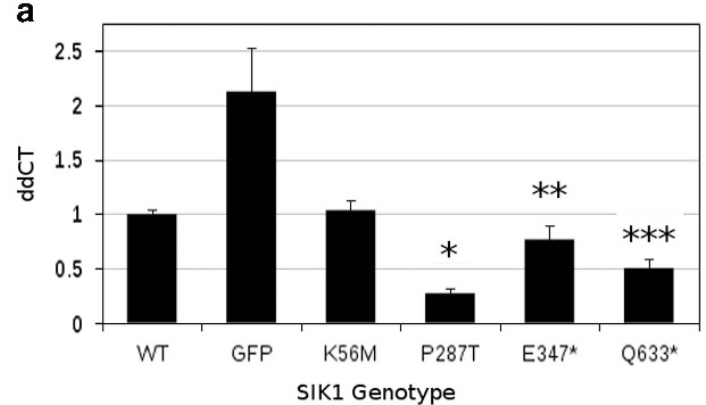

b

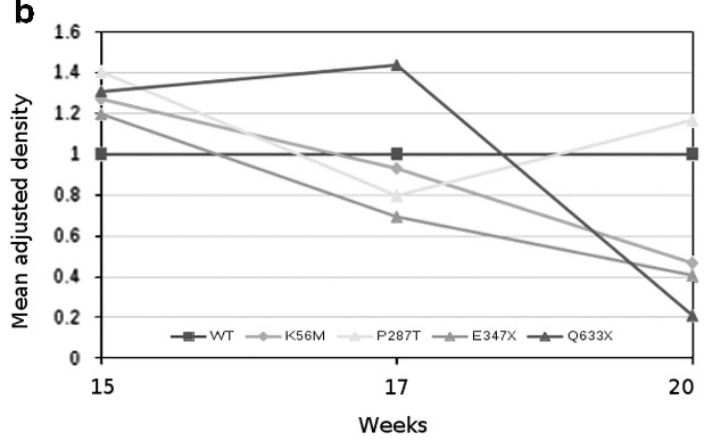

c

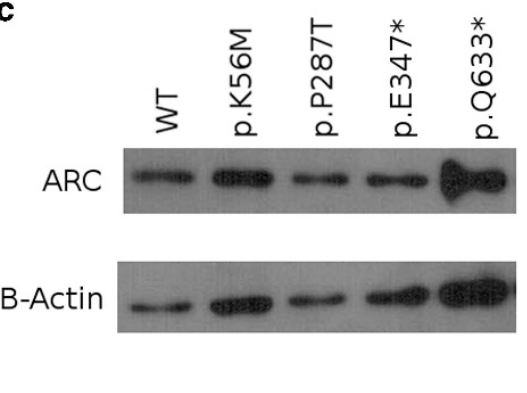

d

ARC

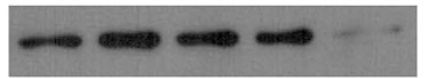

B-Actin

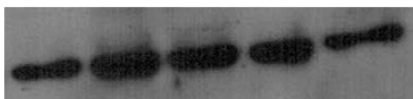

e

ARC

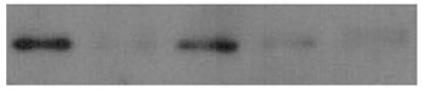

B-Actin

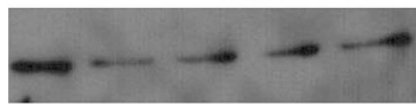

Figure 3 Epilepsy-causing SIK1 sequence variations decreased ARC mRNA and protein levels. Semiquantitative reverse transcriptase-PCR of $A R C$ was significantly reduced in all disease-causing mutants in 20-week fetal-derived primary neurons (a; $\left.{ }^{*} P=0.004 ;{ }^{*} P=0.02 ;{ }^{* *} P=0.0005\right)$ compared with neurons expressing wild-type SIK1. Neurons transfected with GFP were used as a vector control. There was an overall downward trend in ARC protein levels detected by western blot at 15,17 and 20 weeks gestation in all SIK1 mutants compared with wild type. The p.(Glu347*) and p.(Gln633*) sequence variants had significantly $(P=0.05$ and $P=0.007$, respectively) decreased ARC protein levels compared with wild-type neurons at 20 weeks gestation (b). Representative western blots of ARC protein levels in human neurons at 15 weeks (c), 17 weeks (d) and 20 weeks gestation (e) normalized to $\beta$-actin.

The kinase-dead p.(Lys56Met) mutant was associated with a significantly decreased number of proximal branches. The results of the Sholl analysis are summarized in Figures $4 \mathrm{a}-\mathrm{f}$.

We next measured neurite length and number in human neurons expressing wild-type and mutant SIK1 proteins. The missense p.(Pro287Thr) mutant cells had significantly increased neurite number compared with wild-type cells. We did not observe significant differences in neurite number in the truncating p.(Glu347*) and p. $\left(\mathrm{G} \ln 633^{\star}\right)$ cells compared with wild-type cells, although there was a trend toward decreased number of branches. The results of neurite analysis are summarized in Figures $4 \mathrm{~h}-\mathrm{i}$.

\section{DISCUSSION}

This work provides evidence that epilepsy-causing sequence variations in SIK1 have deleterious effects on human neuronal biology. Our data show that the truncating SIK1 sequence variations p.(Glu347*) and p. $\left(\mathrm{Gln} 633^{\star}\right)$ were associated with decreased expression of the transcription factor $M E F 2 C$. This resulted in both decreased $\mathrm{MEF} 2 \mathrm{C}$ protein levels in affected neurons and a decrease in MEF2C transcriptional activity. Furthermore, this disruption of MEF2C activity occurs independently of HDAC5, suggesting that SIK1 may either interact directly with MEF2C or may act through another intermediary. We also show that disruption of MEF2C activity results in the decreased expression of proteins critical for neuronal synapse function and development, prominent among them ARC.

We found previously ${ }^{1}$ that although these SIK1 pathogenic sequence variations occur outside the kinase domain of the protein, and in a cell-free assay mutant SIK1 kinase activity was unaffected, truncated SIK1 mutant proteins did not localize appropriately to the nucleus where HDAC5 phosphorylation occurs. As MEF2C transcriptional activation occurs when HDAC5 is phosphorylated and then exported from the nucleus, ${ }^{3}$ we initially hypothesized that disease-causing sequence variations in SIK1 might affect MEF2C activity by underphosphorylating HDAC5 in the nucleus. Our data here suggest that SIK1 pathogenic sequence variations have a deleterious effect on $\mathrm{MEF} 2 \mathrm{C}$ transcriptional activity separate from the phosphorylation status of HDAC5.

Loss-of-function $M E F 2 C$ sequence variations (including chromosomal 5q14.3 deletions that result in $M E F 2 C$ haploinsufficiency) are associated with a developmental syndrome with common early-life epilepsy, intellectual disability and autistic features. ${ }^{4,5}$ Mef2c-knockout mice have impaired learning with an associated increase in excitatory neuronal synapses. ${ }^{23}$ Postnatal loss of Mef2c resulted in increased spine numbers in hippocampal neurons. ${ }^{24}$ The $\alpha 1 \beta$ isoforms of Mef $2 \mathrm{c}$ are expressed in neurons, ${ }^{25}$ and the $\alpha 1$ exon contains a regulatory D-box (R-X-X-L) and two phosphomotifs, p.(Ser98) and p.(Ser110), that are involved in MEF2C degradation and subsequent cell cycle progression. $^{26}$ It is possible that epilepsy-causing SIK1 sequence variations directly affect these phosphorylation sites of MEF2C, and this is a focus of future experiments.

We did not appreciate disruption of CREB1 transcriptional activity in our studies of neurons expressing mutant SIK1 proteins, although TORC1 and CREB1 regulation is another critical role of SIK1 in neurons. ${ }^{27}$ CREB1-regulated gene expression is a known mediator of 

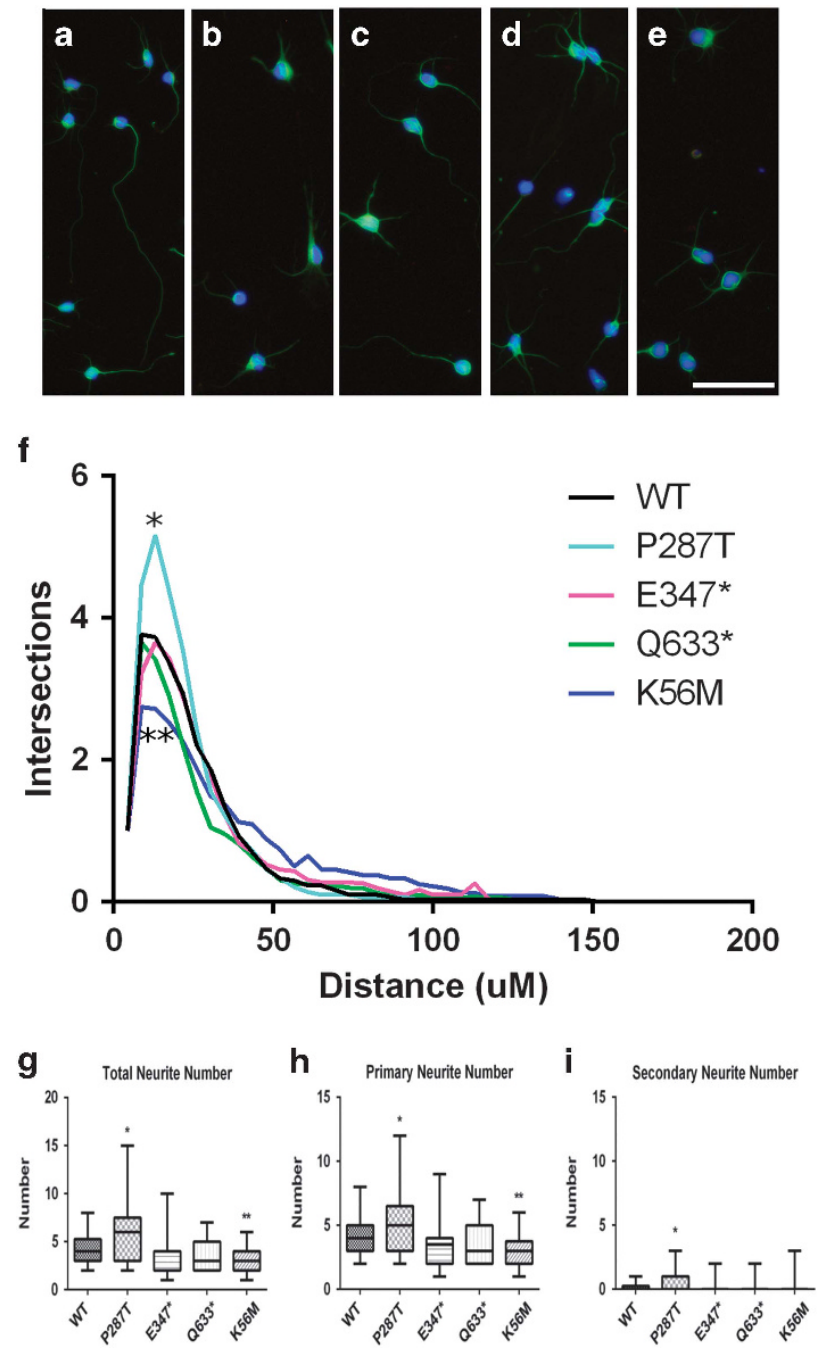

Figure 4 Epilepsy-causing missense SIK1 sequence variation associated with increased proximal neurite branching and increased neurite number. Sholl analysis of neurite branching in neurons infected at 14 weeks and expressing wild-type or mutant SIK1 (a-e; bar $=100 \mu \mathrm{m}$ ) revealed significantly increased proximal intersections in neurons expressing the missense mutant p.(Pro287Thr; 5.1 intersections vs 3.7 intersections; ${ }^{*} P=0.01$ ). The kinase-dead control sequence variant $p$.(Lys56Met) neurons showed significantly fewer proximal intersections (2.7 intersections vs 3.7 intersections; ${ }^{*} P=0.003$ ). No significant differences in intersection number were observed in neurons expressing the truncating SIK1 sequence variants p.(Glu347*) or p.(Gln633*). Neurons expressing the missense p.(Pro287Thr) SIK1 sequence variant also had significantly increased total neurite number (g; $\left.{ }^{*} P=0.016\right)$, increased primary neurite number $\left(\mathbf{h} ;{ }^{*} P=0.046\right)$ and secondary neurite number (i; $* P=0.022$ ) compared with wild type. In contrast, neurons expressing the kinase-dead p.(Lys56Met) SIK1 sequence variant had overall decreased numbers of total neurites $\left(\mathbf{g} ;{ }^{*} P=0.005\right)$ and primary neurites $\left(\mathbf{h} ;{ }^{* *} P=0.004\right)$. No significant differences in neurite number were observed in neurons expressing the truncating SIKI sequence variants.

neuronal dendrite development and long-term potentiation. ${ }^{28}$ SIK1 can have a direct effect on CREB1 transcriptional activity independent of TORC1. ${ }^{29}$ However, our assays of TORC1 and CREB1 in this study did not reveal significant differences from wild-type neurons. It is possible, however, that an effect does exist but our model lacked sensitivity to detect these effects. Given the importance of CREB1 in neuronal development and synapse function, studies of possible effects on this pathway should be included in future studies of SIK1 epilepsy.

We found significant decreases in the $A R C$ gene expression and protein levels in neurons expressing the truncating SIK1 sequence variations. $A R C$ has a critical role during early neuronal development and the MEF2C-ARC-NR4A1 expression pathway is critical for synaptic remodeling, ${ }^{30}$ with ARC specifically having a role in synapse-specific homeostatic plasticity. ${ }^{31}$ Loss-of-function sequence variations in Arc disrupt Notch1 activation, and impair long-term potentiation and longterm depression. ${ }^{32}$ One fascinating aspect of ARC biology is that its mRNA transcripts are transported into dendrites to accumulate nearactivated synapses, where synaptic activity triggers mRNA decay. ${ }^{33,34}$ At excitatory glutamatergic synapses, ARC mediates postsynaptic endocytosis of AMPA-type glutamate receptors. ${ }^{35}$ We therefore expect that decreased ARC expression as a consequence of SIK1 sequence variations would have a disruptive role in these critical neuronal processes.

We also found evidence that mRNA levels of other genes important in synapse activity response, $N R G 1$ and $N R 4 A 1$, were affected by epilepsy-causing SIK1 sequence variations. NRG1 (neuregulin 1) regulates synaptic transmission, in part by controlling glutamate uptake, ${ }^{36}$ and also maintaining GABAergic activity in parvalbumin $(+)$ interneurons. ${ }^{37} \mathrm{NR} 4 \mathrm{Al}$ (Nur77) is an activity-dependent protein that regulates the density and distribution of dendritic spines and excitatory synapses. ${ }^{38}$ In the striatum, synapse remodeling has been shown to be mediated by NR4A1 (Nur77) and ARC, both of which are regulated by MEF2 transcriptional activity. ${ }^{30}$ Abnormalities in these important synaptic activity response proteins suggest that the epilepsy-causing sequence variations in SIK1 result in downstream dysfunction of MEF2C transcription activity, with likely impact on glutamate/ GABA balance, and synapse distribution and remodeling.

Our observations that truncating epilepsy-causing SIK1 sequence variations dysregulate $M E F 2 C$ and $A R C$ allow further questions to be asked regarding the pathogenesis of both the epilepsy and the developmental disorder seen in individuals with SIK1 syndrome. An important role of MEF2C in neurons is to suppress excitatory synapse numbers $^{23}$ in concert with $A R C$ and the metabotropic glutamate receptor 5. ${ }^{39}$ If SIK1 pathogenic sequence variations disrupt this physiologic counterweight to neuronal excitation, it is possible that the threshold for clinical seizures may be lowered. Our studies have established an in vitro model where the electrophysiologic properties of SIK1 mutant neurons may be studied.

Our data demonstrated that the missense p.(Pro287Thr) SIK1 sequence variation was associated with observable abnormalities in neuronal morphology. Overall neurite number was increased in $\mathrm{p}$. (Pro287Thr) SIK1-expressing cells, and there were an increased number of proximal branches. We note that the individual described with the SIK1 p.(Pro287Thr) sequence variation had a severe clinical phenotype, with early-onset infantile epilepsy, persistent burst suppression pattern on EEG and death in infancy. ${ }^{1}$ Although the p.(Pro287Thr)-expressing neurons did not demonstrate the same degree of abnormality in $M E F 2 C$ and $A R C$ expression, it is possible that the severe phenotype associated with this sequence variation is conferred through another mechanism. For example, it is known that SIK1 directly regulates $\mathrm{Na}-\mathrm{K}$ ATPases in the renal proximal tubule and vascular smooth muscle, ${ }^{40,41}$ and dysfunction of that pathway in neurons may be another mechanism where seizures might be generated.

Our studies have several limitations. First, our results were based on overexpression assays in human fetal neurons, and it was not possible to determine the effect of endogenous SIK1 protein on our results. Therefore, it was not possible to conclude whether the SIK1 
pathogenic sequence variations studied cause direct loss of SIK1 function. Our results showing no change in HDAC5 phosphorylation suggest that the SIK1 sequence variations do not have a direct loss-offunction effect. However, our data suggest that these SIK1 sequence variations result in downstream dysregulation of MEF2C signaling.

In conclusion, this study provides the first evidence that epilepsycausing sequence variations in the AMP kinase SIK1 result in significant abnormalities of synapse regulation and neuronal morphology in an in vitro human neuronal model. Truncating SIK1 mutants were associated with widespread disruption of $M E F 2 C$ transcriptional activity, with consequent abnormalities in the expression of the targets ARC, NRG1 and NR4A1. The missense SIK1 sequence variation, associated with a severe human phenotype, was found to also cause abnormal neuronal morphology. These studies extend an important pathway in understanding the pathogenesis of early-life epilepsy, and lay the groundwork for further study of important aspects of these epilepsies with the aim of identifying improved treatment.

\section{CONFLICT OF INTEREST}

The authors declare no conflict of interest.

\section{ACKNOWLEDGEMENTS}

Research reported in this work was supported by the NIH, National Institutes of Neurologic Disorders and Stroke under award numbers K08NS078054 (to ARP), R01NS076617 (to MWH) and the Schmitt Program on Integrative Brain Research (to CP and ARP).

1 Hansen J, Snow C, Tuttle E et al: De novo mutations in SIK1 cause a spectrum of developmental epilepsies. Am J Hum Genet 2015; 96: 682-690.

2 Jagannath A, Butler R, Godinho SIH et al: The CRTC1-SIK1 pathway regulates entrainment of the circadian clock. Cell 2013; 154: 1100-1111.

3 Finsterwald C, Carrard A, Martin J-L: Role of salt-inducible kinase 1 in the activation of MEF2-dependent transcription by BDNF. PLoS One 2013; 8: e54545.

4 Le Meur N, Holder-Espinasse M, Jaillard S et al: MEF2C haploinsufficiency caused by either microdeletion of the $5 q 14.3$ region or mutation is responsible for severe mental retardation with stereotypic movements, epilepsy and/or cerebral malformations. J Med Genet 2010; 47: 22-29.

5 Zweier M, Gregor A, Zweier C et al: Mutations in MEF2C from the 5q14.3q15 microdeletion syndrome region are a frequent cause of severe mental retardation and diminish MECP2 and CDKL5 expression. Hum Mutat 2010; 31: 722-733.

6 Paciorkowski AR, Traylor RN, Rosenfeld JA et al: MEF2C Haploinsufficiency features consistent hyperkinesis, variable epilepsy, and has a role in dorsal and ventral neuronal developmental pathways. Neurogenetics 2013; 14: 99-111.

7 Rodríguez-Tornos FM, San Aniceto I, Cubelos B, Nieto M: Enrichment of conserved synaptic activity-responsive element in neuronal genes predicts a coordinated response of MEF2, CREB and SRF. PLoS One 2013; 8: e53848.

8 Fulp CT, Cho G, Marsh ED, Nasrallah IM, Labosky PA, Golden JA: Identification of Arx transcriptional targets in the developing basal forebrain. Hum Mol Genet 2008; 17: 3740-3760.

9 Strømme P, Mangelsdorf ME, Scheffer IE, Gécz J: Infantile spasms, dystonia, and other $X$-linked phenotypes caused by mutations in Aristaless related homeobox gene, ARX Brain Dev 2002; 24: 266-268.

10 Seltzer LE, Ma M, Ahmed S et al: Epilepsy and outcome in FOXG1-related disorders. Epilepsia 2014; 55: 1292-1300.

11 Shi X-Y, Tomonoh Y, Wang W-Z et al: Efficacy of antiepileptic drugs for the treatment of Dravet syndrome with different genotypes. Brain Dev 2015; 38: 40-46.

12 Milligan CJ, Li M, Gazina EV et al: KCNT1 gain of function in 2 epilepsy phenotypes is reversed by quinidine. Ann Neurol 2014; 75: 581-590.

13 Mikati MA, Jiang Y-H, Carboni M et al: Quinidine in the treatment of KCNT1 positive epilepsies. Ann Neurol 2015; 78: 995-999.

14 Davies SJA, Shih C-H, Noble M, Mayer-Proschel M, Davies JE, Proschel C: Transplantation of specific human astrocytes promotes functional recovery after spinal cord injury. PLoS One 2011; 6: e17328.
15 Proschel C, Stripay JL, Shih C-H, Munger JC, Noble MD: Delayed transplantation of precursor cell-derived astrocytes provides multiple benefits in a rat model of Parkinsons. EMBO Mol Med 2014; 6: 504-518.

16 Lu J, McKinsey TA, Zhang CL, Olson EN: Regulation of skeletal myogenesis by association of the MEF2 transcription factor with class II histone deacetylases. Mol Cell 2000; 6: 233-244.

17 Jeanneteau F, Deinhardt K, Miyoshi G, Bennett AM, Chao MV: The MAP kinase phosphatase MKP-1 regulates BDNF-induced axon branching. Nat Neurosci 2010; 13: 1373-1379.

18 Henny P, Brown MTC, Northrop A et al: Structural correlates of heterogeneous in vivo activity of midbrain dopaminergic neurons. Nat Neurosci 2012; 15: 613-619.

19 Cripps RM, Lovato TL, Olson EN: Positive autoregulation of the myocyte enhancer factor-2 myogenic control gene during somatic muscle development in Drosophila. Dev Biol 2004; 267: 536-547.

20 Ramachandran B, Yu G, Li S, Zhu B, Gulick T: Myocyte enhancer factor 2 A is transcriptionally autoregulated. J Biol Chem 2008; 283: 10318-10329.

21 Parra M: Class Ila HDACs - new insights into their functions in physiology and pathology. FEBS J 2015; 282: 1736-1744.

22 Peebles CL, Yoo J, Thwin MT, Palop JJ, Noebels JL, Finkbeiner S: Arc regulates spine morphology and maintains network stability in vivo. Proc Natl Acad Sci USA 2010; 107: 18173-18178.

23 Barbosa AC, Kim M-S, Ertunc M et al: MEF2C, a transcription factor that facilitates learning and memory by negative regulation of synapse numbers and function. Proc Natl Acad Sci USA 2008; 105: 9391-9396.

24 Adachi M, Lin P-Y, Pranav H, Monteggia LM: Postnatal loss of Mef2c results in dissociation of effects on synapse number and learning and memory. Biol Psychiatry 2015; 80: 140-148.

25 Sekiyama Y, Suzuki H, Tsukahara T: Functional gene expression analysis of tissuespecific isoforms of Mef2c. Cell Mol Neurobiol 2012; 32: 129-139.

26 Badodi S, Baruffaldi F, Ganassi M, Battini R, Molinari S: Phosphorylation-dependent degradation of MEF2C contributes to regulate G2/M transition. Cell Cycle Georget Tex 2015; 14: 1517-1528.

27 Katoh Y, Takemori H, Lin X-Z et al: Silencing the constitutive active transcription factor CREB by the LKB1-SIK signaling cascade. FEBS J 2006; 273: 2730-2748.

28 Li S, Zhang C, Takemori H, Zhou Y, Xiong Z-Q: TORC1 regulates activity-dependent CREB-target gene transcription and dendritic growth of developing cortical neurons. J Neurosci Off J Soc Neurosci 2009; 29: 2334-2343.

29 Katoh Y, Takemori H, Min L et al: Salt-inducible kinase-1 represses cAMP response element-binding protein activity both in the nucleus and in the cytoplasm. Eur $J$ Biochem FEBS 2004; 271: 4307-4319.

30 Tian X, Kai L, Hockberger PE, Wokosin DL, Surmeier DJ: MEF-2 regulates activitydependent spine loss in striatopallidal medium spiny neurons. Mol Cell Neurosci 2010; 44: 94-108.

31 Béïque J-C, Na Y, Kuhl D, Worley PF, Huganir RL: Arc-dependent synapse-specific homeostatic plasticity. Proc Natl Acad Sci USA 2011; 108: 816-821.

32 Alberi L, Liu S, Wang Y et al: Activity-induced Notch signaling in neurons requires Arc/ Arg3.1 and is essential for synaptic plasticity in hippocampal networks. Neuron 2011 ; 69: 437-444.

33 Farris S, Lewandowski G, Cox CD, Steward 0. Selective localization of arc mRNA in dendrites involves activity- and translation-dependent mRNA degradation. J Neurosci Off J Soc Neurosci 2014; 34: 4481-4493.

34 Steward O, Farris S, Pirbhoy PS, Darnell J, Driesche SJV: Localization and local translation of Arc/Arg3.1 mRNA at synapses: some observations and paradoxes. Front Mol Neurosci 2014; 7: 101.

35 Mabb AM, Je HS, Wall MJ et al: Triad3A regulates synaptic strength by ubiquitination of Arc. Neuron 2014; 82: 1299-1316.

36 Yu H-N, Park W-K, Nam K-H et al: Neuregulin 1 controls glutamate uptake by upregulating excitatory amino acid carrier 1 (EAAC1). J Biol Chem 2015; 290 20233-20244.

37 Lu Y, Sun X-D, Hou F-Q et al: Maintenance of GABAergic activity by neuregulin 1-ErbB4 in amygdala for fear memory. Neuron 2014; 84: 835-846.

38 Chen $\mathrm{Y}$, Wang Y, Ertürk $A$ et al: Activity-induced Nr4a1 regulates spine density and distribution pattern of excitatory synapses in pyramidal neurons. Neuron 2014; 83 431-443.

39 Wilkerson JR, Tsai N-P, Maksimova MA et al: A role for dendritic mGluR5-mediated local translation of Arc/Arg3.1 in MEF2-dependent synapse elimination. Cell Rep 2014; 7: 1589-1600

40 Popov S, Silveira A, Wågsäter D et al: Salt-inducible kinase 1 influences $\mathrm{Na}(+), \mathrm{K}$ + -ATPase activity in vascular smooth muscle cells and associates with variations in blood pressure. J Hypertens 2011; 29: 2395-2403.

41 Taub M, Springate JE, Cutuli F: Targeting of renal proximal tubule Na, K-ATPase by saltinducible kinase. Biochem Biophys Res Commun 2010; 393: 339-344.

Supplementary Information accompanies this paper on European Journal of Human Genetics website (http://www.nature.com/ejhg) 